\title{
IL POSITIVISMO NELL'EVOLUZIONE DEL PENSIERO DI NORBERTO BOBBIO
}

\section{POSITIVISM IN THE EVOLUTION OF THE THINKING OF NORBERTO BOBBIO}

\author{
Mario G. Losano*
}

RIASSUNTO: Attraverso l'analisi del contesto intelettuale e dei testi dell'autore, questo articolo studia il percorso del pensiero di Norberto Bobbio e i suoi rapporti con il positivismo.

PAROLE CHIAVE: Positivismo. Il pensiero di Norberto Bobbio. Teoria del diritto.

ABSTRACT: Through the analysis of the intellectual context and of the texts of the author, this article studies the trajectory of the thinking of Norberto Bobbio and its relations with positivism.

KEYWORDS: Positivism. The thinking of Norberto Bobbio. Legal theory.

Indice: 1. La "Scuola di Torino": "scuola" in che senso? - 2. Come nasce la filosofia del diritto a Torino: Pietro Luigi Albini. - 3. Un secolo di filosofia del diritto a Torino: 1872-1972. - 4. Bobbio e il positivismo giuridico di Hans Kelsen. - 5. Il diritto come ordinamento: una teoria generale. - 6 . Positivismo e giusnaturalismo in Norberto Bobbio: a) la definizione del positivismo giuridico nei tre corsi; b) la revisione critica del positivismo giuridico nei due volumi. - 7. Dal positivismo giuridico all'apertura al funzionalismo. - 8. Dal positivismo di Bobbio a una teoria "veramente" generale del diritto.

\section{§1. LA “SCUOLA DI TORINO”: “SCUOLA” IN CHE SENSO?}

È sempre difficile individuare una scuola, massime la "Scuola di Torino": agli occhi dell'osservatore, essa prende forma quando l'attenzione si concentra sugli elementi che accomunano gli studiosi che potrebbero comporla, ma si dissolve quando invece egli guarda alle loro differenze.

Quando oggi si parla di “Scuola di Torino” si intende in generale il gruppo di studiosi che si è formato intorno a Bobbio negli anni in cui insegnò filosofia del diritto, cioè fino al $1972^{1}$, anche se dopo quella data si formò intorno a lui un gruppo di politologi. In queste pagine riprendo alcune

\footnotetext{
* Dottore honoris causa presso l'Università di Hannover (Germania); della Repubblica, Montevideo (Uruguay); Carlos III, Madrid (Spagna); Professore onorario presso l'Università Federale di Pernambuco (Brasile). Professore Emerito di Filosofia del Diritto e Introduzione alla Informatica Giuridica presso la Facoltà di Giurisprudenza dell'Università del Piemonte Orientale, Alessandria (Italia). Professore presso la Scuola di Dottorato dell'Università degli Studi di Torino (Italia).E-mail: mario_losano@yahoo.it

${ }^{1}$ Unzueta, María Ángeles Barrère, La escuela de Bobbio. Reglas y normas en la filosofía jurídica italiana de inspiración analítica, Tecnos, Madrid 1990, p. 35; contiene un bilancio degli anni Settanta (p. 76), ma l’intero volume è importante per un esame approfondito di una ‘scuola' in divenire.
} 
delle considerazioni che ho svolto in modo più organico in una biografia culturale di Norberto Bobbio, ancora inedita: mi scuso quindi se vari temi qui affrontati non presentano lo sviluppo che meriterebbero. Spero tuttavia che risulti visibile la linea evolutiva che la filosofia del diritto ha avuto a Torino nel corso di oltre un secolo e mezzo.

A Torino infatti, prima di Bobbio, la filosofia del diritto venne introdotta da Pietro Luigi Albini, studioso fino a qualche anno fa piuttosto trascurato, e coltivata poi da Giuseppe Carle, maestro di Gioele Solari, e da Gioele Solari, maestro quest'ultimo di Norberto Bobbio. La continuità di questo gruppo può giustificare l’attribuirgli la denominazione di “scuola”.

Dunque il giovane Bobbio, studente di giurisprudenza all’Università di Torino, fu allievo di Gioele Solari. Il gruppo che si formò intorno a Gioele Solari tra le due guerre mondiali sentì una comune appartenenza e la espresse nel volume che avrebbe dovuto salutare gli ottant'anni del suo maestro, e che invece ne commemorò la scomparsa. "Attorno a lui, - così si legge nella presentazione, - e per la serietà a cui aveva ispirato la sua vita e per l'esempio di dirittura scientifica ch'egli offriva e per il calore comunicativo che emanava dalla sua persona, si è costituita, cosa rara, una scuola. Molti furono i giovani che, soprattutto nel trentennio del suo magistero torinese (19181948), si volsero, da lui spronati e diretti, agli studi scientifici e, conseguita la laurea, li hanno proseguiti nei diversi campi dal maestro coltivati, diventando a loro volta, o avviandosi a diventare, docenti universitari”,2.

Ma questi docenti trovarono la materia “filosofia del diritto” già incardinata come materia obbligatoria nell'ordinamento degli studi dell’Università di Torino. Per risalire all'origine di questo insegnamento bisogna ritornare all’Ottocento e a Pietro Luigi Albini.

\section{§2. COME NASCE LA FILOSOFIA DEL DIRITTO A TORINO: PIETRO LUIGI ALBINI}

Il moderno insegnamento della filosofia del diritto nell'università di Torino iniziò con Pietro Luigi Albini (1807-1863), professore, avvocato e - per breve tempo - politico negli anni che portarono all'unità d'Italia (LOSANO, 2013) ${ }^{3}$. Il suo interesse per la storia del diritto e per la filosofia del diritto è strettamente collegato con il suo insegnamento dell'”enciclopedia giuridica” nell’università di Torino.

\footnotetext{
${ }^{2}$ BALBO et al., 1954. La citazione riportata nel testo è a p. 7. L'elenco dei “discepoli” fornisce, se non un quadro oggettivo, almeno un autoritratto autentico della scuola raccolta intorno a Solari; autentico, ma non completo: "non tutti coloro che sono stati invitati, - avverte infatti la prefazione, - hanno potuto inviare il loro contributo. E forse alcuni [...] saranno stati dimenticati” (p. 8).

${ }^{3}$ Seguita (sempre presso l'Accademia delle Scienze di Torino) da una seconda Memoria: I carteggi di Pietro Luigi Albini con Federico Sclopis e Karl Mittermaier (1839-1856). Alle origini della filosofia del diritto a Torino.
} 
Due contatti scientifici ebbero particolare importanza nella sua vita: quello con Federico Sclopis e quello con Karl Mittermaier, con i quali ebbe un carteggio in corso di stampa presso l’Accademia delle Scienze di Torino (LOSANO, 2014 [in stampa]). Sclopis fu il suo protettore nella Torino capitale sabauda, mentre Mittermaier fu il corrispondente che lo rese noto fuori dai confini piemontesi e che gli fornì le informazioni sulla filosofia del diritto tedesca e sull’organizzazione delle università tedesche. Attraverso queste notizie e grazie alla sua partecipazione alle commissioni per la riforma dell'insegnamento del diritto, Albini introdusse il modello tedesco nell’Università di Torino. Il Piemonte, infatti, era tradizionalmente ispirato al modello francese, ma in quegli anni stava scoprendo quello tedesco: le vicende risorgimentali lo avevano allontanato dalla Francia, mentre dopo la battaglia di Sedan ascendeva l'astro tedesco.

Le principali opere di Albini si possono organizzare in tre filoni che si intersecano costantemente nella sua vita: la filosofia del diritto, l'enciclopedia del diritto e la storia del diritto e, infine, le sue prese di posizione sui diritti e sulle libertà del cittadino, in particolare sulla pena di morte e sulla libertà d'insegnamento. Albini era aperto alle idee liberali, e il suo liberalismo moderato si riflette tanto nei suoi scritti quanto nella sua breve attività parlamentare.

A fondamento dell'intera sua opera sta una visione del diritto come prodotto dello "spirito del popolo", secondo gli insegnamenti della Scuola Storica tedesca: "Il diritto si sviluppa nel popolo e per mezzo del popolo come la sua lingua” (e quindi lo strumento più adatto ad esprimere ogni concetto è "la lingua nazionale") (ALBINI, 1839, p. 327). Anche più tardi ritorna questa convinzione: "Il diritto è per lo più una conseguenza della condizione e delle vicende politiche di un popolo, e l'espressione del suo stato intellettuale e morale” (ALBINI, 1847, Prefazione, p. XI). L'attività di giurista pratico porta Albini ad associare questa convinzione all'applicazione e all'innovazione del diritto positivo: quindi per Albini diritto e politica costituiscono un binomio inscindibile. A questo duplice indirizzo fa riferimento anche il titolo della sua prima opera del 1839 (ALBINI, 1839). L’intera scienza giuridica comprende per lui la filosofia, la storia e la pratica del diritto - e questa tripartizione è richiamata all'inizio del volume attraverso una citazione di Vico (1720, p. VII).

Un ventennio dopo la riforma universitaria piemontese l’insegnamento della filosofia del diritto si era ormai consolidato. Nel 1857 Albini pubblicò per i suoi studenti una "semplice esposizione sommaria dei principi cardinali della dottrina filosofica del diritto”, tenendosi lontano dalle "teorie astruse" che "poco o nulla utili riescono nelle pratiche applicazioni della vita civile" 
(ALBINI, 1857, p. V et seq.) ${ }^{4}$ : come nelle altre sue opere, la pratica del diritto è al centro delle sue ricerche e del suo insegnamento. Questa finalità didattica si riflette nella bibliografia che conclude il volume e che rappresenta quasi la biblioteca ideale dell'autore. La sua impostazione riflette il duplice interesse analitico e storico che caratterizza tutta l’opera di Albini. Infatti la Biblioteca scelta di filosofia del diritto elenca i sistemi giusfilosofici esemplari che egli addita agli studenti, mentre l'evoluzione delle idee giusfilosofiche è illustrata nei titoli Sulla storia della filosofia del diritto.

Con le sue opere e con la sua attività di riformatore degli studi universitari piemontesi Albini si colloca così alle origini della scuola di filosofia del diritto che si sviluppò poi a Torino con Giuseppe Carle, Gioele Solari e Norberto Bobbio.

\section{§3. UN SECOLO DI FILOSOFIA DEL DIRITTO A TORINO: 1872-1972}

Se scuola c’è, è nella continuità di serietà didattica e d’impegno civile e politico. Nell'arco di un secolo - dal 1872 al 1972 - tre nomi hanno illustrato quella cattedra: Giuseppe Carle, che insegnò dal 1872 al 1917; il suo allievo Gioele Solari, che insegnò dal 1918 al 1942 e dal 1945 al 1948; e Norberto Bobbio, allievo di Solari, che insegnò dal 1944 al 1945 e, poi, dal 1948 al 1972 $(\text { ORECCHIA, 1967) })^{5}$ (mentre dopo il 1972 Bobbio insegnò filosofia della politica presso la facoltà torinese di scienze politiche). In un secolo esatto di insegnamento della filosofia del diritto, accanto a questi tre nomi soltanto altri due compaiono come incaricati dell'insegnamento per brevi periodi alla fine delle due guerre mondiali: il processualista e penalista Cesare Civoli ${ }^{6}$, per l'anno 1917-18, e il filosofo Augusto Guzzo ${ }^{7}$ nel 1944-45.

Il senso della continuità si riscontra anche nel fatto che Solari dedicò un libro a Carle (SOLARI, 1926) $^{8}$, e Bobbio vari scritti a Solari, fra cui una monografia subito dopo la sua morte

\footnotetext{
${ }^{4}$ Questo e altri scritti di Albini sono digitalizzati open access presso la Bayerische Staatbibliothek di Monaco di Baviera (https://www.bsb-muenchen.de/literatursuche/).

${ }^{5}$ Cfr. anche BOBBIO, 1997, p. 168.

${ }^{6}$ Luigi Cesare Civoli (Genova, 16 dicembre 1861 - Cavi di Lavagna, 28 luglio 1930) fu ordinario di diritto e procedura penale nelle Università di Pavia e, poi, di Genova: Falchi, A., Cesare Civoli. Necrologio, "Rivista italiana di diritto penale”, 1931, n. 3-4, pp. 342-344; Orecchia, cit., p. 105. Non registrano sue opere di filosofia del diritto né Orecchia né il Clio (Catalogo dei libri italiani dell'Ottocento (1801-1900), Editrice Bibliografica, Milano 1991, 19 voll.)

${ }^{7}$ Augusto Guzzo (Napoli, 24 gennaio 1894 - Torino, 23 agosto 1986) fu ordinario di filosofia teoretica all’Università di Torino: Plebe, Armando et al., Augusto Guzzo, Edizioni di "Filosofia”, Torino 1964, 127 pp. (seconda edizione); Solari, Gioele, La dottrina della giustizia nel sistema della moralità di Augusto Guzzo, "Rivista di filosofia”, 1951, n. 4, pp. 378-398. Guzzo scrisse il necrologio di Gioele Solari in "Filosofia", 1952, pp. 472-473 e, inoltre, Incontri con Gioele Solari, in Gioele Solari. 1872-1952. Testimonianze e bibliografia nel centenario della nascita, Memorie dell’Accademia delle Scienze, Classe di Scienze Morali, Storiche e Filologiche, Torino 1972, pp. 1-8.

${ }^{8}$ Anche separatamente: Bocca, Torino 1928, 191 pp.
} 
(BOBBIO, 1952-53). Per brevità non mi soffermo qui su questi predecessori di Norberto Bobbio, rinviando ai loro scritti appena citati e al mio saggio in cui ho concentrato l'attenzione su queste tre figure che rappresentano una secolare continuità di insegnamento: in esso ho esaminato soprattutto gli elementi culturali e personali su cui si fonda questa continuità (LOSANO, 1999).

\section{§4. BOBBIO E IL POSITIVISMO GIURIDICO DI HANS KELSEN}

"Se dovessi fissare una data da cui far iniziare le opere della [mia] maturità, - scrive Bobbio, - sceglierei il 1949, anno in cui pubblicai in Italia un'analisi e un commento alla Teoria generale del diritto di Francesco Carnelutti” (BOBBIO, 1980, p. 10) ${ }^{9}$. Quella data segna anche la “conversione” di Bobbio al normativismo, perché prese posizione a favore della teoria normativistica di Kelsen, criticata appunto da Francesco Carnelutti. Questo importante giurista e avvocato aveva insegnato a Padova dal 1915 al 1936, mentre Bobbio fu chiamato a quell’università nel 1938, quando Carnelutti era già passato all’Università Statale di Milano: fra i due non vi fu quindi un contatto diretto. Però nel 1946 Carnelutti aveva pubblicato la "seconda edizione interamente nuova” della sua discussa teoria generale del diritto ed essa attirò l'attenzione di Bobbio (CARNELUTTI, 1946) ${ }^{10}$, che di Carnelutti criticò la dottrina imperativistica del diritto, mentre si pronunciò a favore della dottrina pura del diritto: per questo il suo saggio del 1949 segna la sua "conversione” a Kelsen.

Il pensiero di Kelsen era noto in Italia sino dagli anni Venti, anche perché una rivista legata al movimento fascista aveva pubblicato alcuni suoi saggi sulla democrazia, sia pure accompagnandoli con una nota che ne sottolineava l'importanza scientifica ma che, al tempo stesso, prendeva le distanze dalla loro posizione ideologica, favorevole alla deprecata democrazia ${ }^{11}$. Per

\footnotetext{
${ }^{9}$ Una precisa ricostruzione del pensiero teorico-giuridico di Bobbio è contenuta nella presentazione a quel volume: Alfonso Ruiz Miguel, Bobbio y el positivismo jurídico italiano, pp. 15-58 e, soprattutto, nel libro che fa di Alfonso Ruiz Miguel il "bobbiologo" en titre non solo in Spagna: Filosofía y derecho en Norberto Bobbio, Centro de Estudios Constitucionales, Madrid 1983, 509 pp.

${ }^{10}$ Bobbio espresse la sua preferenza per la teoria di Kelsen nell'ambito di un'analisi generale di quest'opera: Francesco Carnelutti, teorico generale del diritto, “Giurisprudenza Italiana”, 1949, col. 113-127; l'anno successivo Bobbio ritornò sulla sua concezione della teoria generale del diritto, limitando il riferimento a Carnelutti alla prima e all'ultima pagina del saggio Filosofia del diritto e teoria generale del diritto, in Scritti in onore di Francesco Carnelutti, Cedam, Padova 1950, vol. 1, pp. 43-69. Questi due saggi - che possono essere considerati complementari - sono anche in Bobbio, Norberto, Studi sulla teoria generale del diritto, Giappichelli, Torino 1955a, pp. 1-26 e 27-52.

${ }^{11}$ Su questo primo contatto del democratico Kelsen con l'Italia fascista, cfr. Losano, Mario G., Tra democrazia in crisi e corporativismo in ascesa: il primo libro italiano di Hans Kelsen, in Hans Kelsen - Arnaldo Volpicelli, Parlamentarismo, democrazia e corporativismo. Prefazione e cura di Mario G. Losano, Nino Aragno Editore, Torino 2012, pp. 7-79. In generale: Losano, Mario G., Reine Rechtslehre in Italien, in Der Einfluß der Reinen Rechtslehre auf die Rechtstheorie in verschiedenen Ländern, Manz, Wien 1978b, pp. 151-79; ripreso in La fortuna di Hans Kelsen in
} 
distanziarsi da questa collocazione fuorviante, nel 1933 Kelsen insistette con Giorgio Del Vecchio affinché il saggio che sintetizzava la sua teoria pura del diritto venisse pubblicato in Italia sulla “Rivista Internazionale di Filosofia del Diritto”. Nel 1933, infatti, Kelsen si preparava al secondo esilio (che lo avrebbe portato da Colonia a Ginevra) inviando ai colleghi stranieri più in vista una magistrale sintesi della sua teoria giuridica, nella speranza che essa contribuisse ad aprirgli le porte di un’università in cui trovare accoglienza.

Il manoscritto di Kelsen giunse così nelle mani di Del Vecchio, che ne affidò la traduzione a Renato Treves, fraterno amico di Bobbio e anch'egli allievo di Solari: Treves infatti aveva incontrato personalmente Kelsen in Germania, nel suo viaggio di studio in Germania nel 1932, con Bobbio e Geymonat (che diverrà un importante filosofo della scienza). Quel manoscritto costituì la prima edizione dell'opera fondamentale di Kelsen e quella traduzione divenne nel 1934 la prima edizione italiana della Dottrina pura del diritto. La Bibbia del positivismo giuridico più rigoroso entrava così in Italia in stretto contatto con la Scuola di Torino. "Da Treves si può far cominciare la fortuna di Kelsen in Italia”, scriverà Bobbio nella prefazione alla raccolta dei propri saggi kelseniani (BOBBIO, 1992, p. 5). A questa fortuna contribuì molto anche Bobbio, ma soprattutto a partire dal 1954. I suoi studi giusfilosofici anteriori affrontavano infatti temi estranei al positivismo kelseniano (come l'analogia) o assumevano posizioni antipositivistiche (come nell'analisi della consuetudine).

La “conversione” del 1949 coincise anche con il bisogno di rinnovamento che pervase l'Italia dopo la fine della guerra e con l'insoddisfazione di Bobbio nei riguardi della filosofia puramente speculativa, cui contrapponeva la filosofia positiva di Carlo Cattaneo ${ }^{12}$. Iniziava così il periodo - destinato a durare circa trent'anni - in cui Bobbio si accostò criticamente al positivismo giuridico di Kelsen e contribuì in modo decisivo a diffondere la dottrina pura del diritto in Italia.

Nella critica a Carnelutti Bobbio chiarisce anzitutto la sua visione dei rapporti tra filosofia del diritto e teoria generale del diritto, ritornando così ai temi presenti nella sua riflessione sin dalla tesi di laurea con Gioele Solari. Ma da essa sono ormai passati una ventina d'anni e si avverte la maturazione scientifica di Bobbio: mentre là seguiva l'idealismo dominante, qui ricerca una sua via e la trova nel normativismo kelseniano. Quindi, tracciando una biografia culturale di Bobbio, è ora

\footnotetext{
Italia, “Quaderni fiorentini per la storia del pensiero giuridico moderno”, 1979, n. 9, pp. 465-500, e in Losano, Mario G., Forma e realtà in Kelsen, Comunità, Milano 1981, pp. 179-212.

12 “Parlo di 'conversione' perché soltanto così spiego, da un lato, l’oblio in cui ho lasciato sprofondare i miei scritti giuridici precedenti e, dall'altro, la confessione più volte fatta, secondo cui alla rottura violenta col passato avvenuta nella storia del nostro paese tra il 1934 e il 1946 ha corrisposto una frattura nel corso della mia vita privata e pubblica, intellettuale e morale. Incepit vita nova”: Bobbio, Diritto e Potere, cit., Napoli 1992, p. 7.
} 
rilevante ricostruire che cosa egli intendesse per teoria generale del diritto e per filosofia del diritto, lasciando in disparte le critiche a Carnelutti, che, pur interessanti, si appuntano su un'opera ormai consegnata al passato.

Nella raccolta dei saggi che segnano il passaggio al normativismo è possibile individuare l'architettura fondamentale delle concezioni di Bobbio filosofo del diritto, che egli è andato affinando per tutta la vita pur non avendo mai voluto raccoglierle in un unico "sistema". Nel volume del 1955 sulla teoria generale del diritto la posizione di Bobbio va delineandosi per contrasto con le dottrine di altri teorici del diritto: anzitutto con quella di Carnelutti e, poi, con quelle di Alessandro Levi, di Paul Roubier, di Jean Dabin e di Jean Haesaert, mentre il saggio su La teoria pura del diritto e i suoi critici (pubblicato nel 1954 e riportato anche in apertura del volume Diritto e potere del 1992) segna il definitivo consolidarsi delle sue concezioni nella direzione giuspositivistica.

Nel 1949-50, precisando le linee generali della propria visione del mondo giuridico, Bobbio aveva così definito la sua posizione: "Noi sosteniamo che la teoria generale sia una disciplina formale, ma ciò non di meno sia una disciplina scientifica"; quindi essa "appartiene alla scienza e non alla filosofia", e inoltre "non è ricerca di contenuti ma indagine formale”. Una “scienza formale del diritto” (come propongono Roguin, Somló o Kelsen) “altro non è se non quello che comunemente si chiama di solito teoria generale del diritto” (BOBBIO, 1955a, p. 33 e p. 37). In conclusione, "in quanto ricerca degli elementi costitutivi strutturali del diritto, la teoria generale è una teoria del diritto positivo e vale nell'ambito di un determinato sistema” (BOBBIO, 1955a, p. 40). Quindi “si può parlare di una teoria generale del diritto italiano, del diritto internazionale, ecc." ed eventualmente di lì risalire a "uno studio sistematico dell'ordinamento giuridico in generale", cioè a una “teoria generale dell'ordinamento giuridico e in questo caso, e soltanto in questo caso, la denominazione di teoria generale avrà lo stesso significato che nell'espressione teoria generale dello Stato” (BOBBIO, 1955a, p. 8 et seq.).

Rispetto a questa definizione, due sono le possibili “deviazioni” della teoria generale del diritto: o verso le discipline giuridiche particolari, "di cui finirebbe per essere soltanto una generalizzazione o una tipizzazione" (secondo che al termine "generale" si attribuisca il significato di "universale" ovvero di "ideal-tipico"), o verso la filosofia del diritto (se intende indicare un valore cui il diritto deve ispirarsi, se cioè - come ogni filosofia - "contiene una presa di posizione dell’uomo di fronte al mondo") (BOBBIO, 1955a, p. 7). Per Bobbio la teoria generale di Carnelutti è una "teoria generale del diritto positivo italiano", che però devia "esclusivamente nella direzione della filosofia” (BOBBIO, 1955a, p. 10). Carnelutti aveva anzi dedicato a questa sua concezione 
della filosofia del diritto (il cui compito sarebbe quello di introdurre l'etica nell'economia) un'apposita esposizione nell'Introduzione alla prima edizione della sua opera, ma - di fronte alle critiche quasi unanimi - l'aveva poi abolita nella seconda edizione (CARNELUTTI, 1940, pp. 55$57)^{13}$. Tuttavia quei principi filosofici continuavano a costituire il fondamento dell’intera opera.

Inoltre, fra le varie teorie del diritto, Carnelutti si schiera a favore del diritto come complesso di comandi, cioè a favore della teoria imperativistica, che secondo Bobbio "s’identifica logicamente con la teoria statualista del diritto": "le due teorie si implicano a vicenda" e sono due aspetti che riflettono "il processo di formazione dello Stato moderno" (BOBBIO, 1955a, p. 15). Per entrambe l'unica fonte del diritto è lo Stato. La teoria imperativista è perciò storicamente condizionata, non ha cioè valore assoluto, e quindi non spiega tutti i fenomeni giuridici. In particolare, Bobbio analizza le carenze della teoria imperativista nei riguardi del diritto internazionale e della consuetudine. Però a questo punto, più che sulla teoria accettata da Carnelutti, è opportuno soffermarsi sulla nuova teoria che richiama l'attenzione di Bobbio.

La teoria normativistica, "relegando il momento del comando tra i fenomeni psicologici o sociologici”, si concentra "su ciò che propriamente cade sotto l'indagine del giurista, cioè la regola" e tende "ad ovviare agli inconvenienti già rilevati della teoria imperativistica, fissando la propria attenzione su ciò che accomuna i vari territori dell'esperienza giuridica, ma non su quello che li divide, e ponendo le basi per superare definitivamente con un taglio netto la fonte storica e ideologica di tutti gli inconvenienti e le ambiguità: l’identificazione fra diritto e Stato” (BOBBIO, 1955a, p. 20). È a questo punto, ormai alla fine del saggio del 1949, che Bobbio prende posizione a favore della dottrina pura del diritto.

Carnelutti, cui l'autostima non faceva certo difetto, si sbarazza del normativismo con un riconoscimento generico, ma senza una vera e propria critica. Nelle poche pagine in cui affronta esplicitamente la teoria normativistica, il tono è più larvatamente polemico che costruttivamente critico, a partire dal momento in cui, nell'introduzione, attribuisce l'ostilità verso la propria teoria generale al fatto di non portare "il suggello della scuola di Vienna o di Berlino" (CARNELUTTI, 1946, p. V).

\footnotetext{
${ }^{13}$ Considerazioni filosofiche, cui Carnelutti era particolarmente affezionato, vennero però rielaborate e pubblicate "in un libercolo a sé, pubblicato oltre due anni fa e perciò, rispetto al mio pensiero, rapidamente invecchiato”: CARNELUTTI, 1946, cit., p. VI. Carnelutti si riferisce alla sua Introduzione allo studio del diritto, Foro Italiano, Roma 1943, 88 pp.: “più d’un capitolo di essa, specialmente verso la fine, dovrebb’essere rifatto” (Teoria generale del diritto [1946], cit., p. 5, in nota).
} 
Per Carnelutti una teoria va costruita tanto sull’analisi della natura del dato, oggetto del suo studio, quanto, in un successivo momento, sulla sintesi dei risultati raggiunti. Questa concezione è ben diversa da quella kelseniana avversata da Carnelutti, che sostiene:

Certe correnti e certi periodi del pensiero giuridico sono contrassegnati dalla tendenza al prevalere dell'analisi, che cela in sé un grave pericolo per lo sviluppo della scienza del diritto. Questo è, in particolare, il difetto di una moderna scuola giuridica, che ha conquistato una grande fama, in buona parte per le sue reali benemerenze; essa medesima si è data il nome di teoria pura del diritto; il suo carattere sta in un possente sforzo analitico e la sua formula fondamentale nella distinzione tra la scienza e la politica del diritto. [Questa teoria è] "un contributo molto serio alla conoscenza del dato" [, ma] "il suo torto metodologico consiste in uno squilibrio tra il momento analitico ed il momento sintetico dell'indagine" [. Il prevalere dell'analisi] "ha certo giovato alla profondità ma ha nociuto alla compiutezza del conoscere” (CARNELUTTI, 1946, p. 3, corsivi di Carnelutti).

Per Bobbio questo sommario riferimento allo "sforzo analitico” e alla “distinzione fra la scienza e la politica del diritto" è "manifestamente generico e inadatto a individuare una scuola piuttosto che un'altra” (BOBBIO, 1955a, p. 21). Non risulta più convincente neppure l'altro passo di Carnelutti che individua un "errore della concezione normativa del diritto" ${ }^{14}$ nella confusione tra norma e legge giuridica. Bobbio "ha l'impressione che la citazione sia male appropriata, o comunque fuor di proposito, a meno che non si riduca questo addotto contrasto ad una mera questione di parole” (BOBBIO, 1955a, p. 22). Carnelutti risponderà a queste critiche nel 1951 nella prefazione alla terza edizione della sua Teoria generale del diritto e ad esse Bobbio farà seguire una breve replica nel $1955^{15}$.

In questo dibattito Bobbio ha messo a fuoco la fecondità scientifica della teoria pura del diritto: "Se dovessi definire brevemente il significato della teoria pura del diritto dal punto di vista metodologico, direi che essa ci ha insegnato soprattutto una cosa: che la scienza giuridica diventa sempre più scienza rigorosa, cioè si risolve in un sistema di sapere avente validità teoretica, quanto più riesce ad espellere dalla sua ricerca i problemi scientificamente insolubili e i problemi giuridicamente irrilevanti” (BOBBIO, 1955a, p. 23, corsivi di Bobbio). I primi sono quelli che non si risolvono con il procedimento scientifico, ma, per esempio, ricorrendo al pensiero magico o mistico, mentre i secondi usano il procedimento scientifico, proprio però d'una scienza diversa dal diritto. Alla teoria pura del diritto Bobbio attribuisce perciò il merito di aver introdotto nella scienza giuridica la scientificizzazione (cioè l'eliminazione degli elementi non scientifici) e la purificazione (cioè l'eliminazione degli elementi non giuridici).

\footnotetext{
${ }^{14}$ Il testo di Bobbio rinvia a CARNELUTTI, 1946, cit., p. 83, però il passo citato da Bobbio non si trova a questa pagina.

${ }^{15}$ Rispettivamente, CARNELUTTI, 1951, pp. XV et seq.; BOBBIO, 1955a, cit., p. 25 et seq.
} 
A partire dal 1949 Bobbio aderì alla teoria formale di Kelsen e in un articolo del 1954 (BOBBIO, 1954) ne prese "calorosamente, direi quasi baldanzosamente, le difese dai suoi detrattori” (BOBBIO, 1992, P. 8), che sono soprattutto i sociologi e i giusnaturalisti. A quel panorama delle critiche alla dottrina pura del diritto seguì nel 1973 una ricostruzione di quella teoria (BOBBIO, 1973), perché in quell'anno la morte di Hans Kelsen indusse Bobbio a tracciare un bilancio critico della dottrina pura del diritto ${ }^{16}$. Questo bilancio apprezza i risultati raggiunti dal positivismo giuridico e, al tempo stesso, prospetta gli obiettivi che, dopo di esso, deve perseguire una teoria del diritto che voglia tenere il passo con i tempi. Infatti quel necrologio, con il rinnovato titolo in cui Bobbio collega il concetto di struttura a quello di funzione, rappresenta il completamento e il superamento della ricezione kelseniana in Bobbio: sull'abbinamento di questi due concetti ritornerò nei $\S \S 6$, b e 7.

Tuttavia il positivismo kelseniano ispirò a Bobbio anche un’opera unitaria, che però conobbe un curioso percorso editoriale, perché nacque in Colombia - all’insaputa di Bobbio dall'unione di due sue dispense: Bobbio stesso accettò poi di farne anche un'edizione italiana $(B O B B I O, 1987)^{17}$. Su questa edizione torneremo nel prossimo paragrafo. Le due dispense in questione sono il frutto, negli anni Cinquanta, di due corsi di Bobbio ("più volte da me ripetuti”) sulla teoria della norma (BOBBIO, 1958) ${ }^{18}$ e sulla teoria dell’ordinamento (BOBBIO, 1960) ${ }^{19}$, “che giustamente sono stati definiti di netta ispirazione kelseniana” (BOBBIO, 1992, p. 8). In Bobbio, il frutto più cospicuo del periodo kelseniano fu la visione del diritto non come norma, ma come ordinamento di norme, che egli espose nei corsi degli anni accademici 1957-58 e 1959-60. In essi la

\footnotetext{
${ }^{16}$ Nella "Rivista internazionale di filosofia del diritto" il saggio di Bobbio venne pubblicato come necrologio, col titolo Hans Kelsen e con una breve nota editoriale omessa nelle ristampe successive ("Rivista internazionale di filosofia del diritto”, 1973, pp. 425-449); nel volume del 1977, Dalla struttura alla funzione, quel saggio venne ripubblicato col titolo Struttura e funzione nella teoria del diritto di Kelsen (pp. 187-215; e tra le fonti, a p. 12, lo si indica come tratto dalla "Rivista internazionale di filosofia del diritto", 1973, pp. 426-449 [dove però quel 426 deve leggersi 425]); nel volume del 1992, Diritto e Potere, lo stesso scritto venne ripreso con lo stesso titolo (pp. 65-87; e tra le fonti, a p. 215, lo si indica come tratto dalla "Rivista internazionale di filosofia del diritto", 1973, però erroneamente alle pp. 187-215). In conclusione, Dalla struttura alla funzione, 1977, riporta la fonte in modo corretto (salvo quel 426, invece di 425); il volume Diritto e Potere, 1992, riporta la fonte scambiando però il numero delle pagine della rivista con quello del volume del 1977 (indica cioè pp. 187-215, invece di pp. 425-449). Inappuntabile Carlo Violi, che nella sua bibliografia di Bobbio indica il corretto numero di pagine della "Rivista internazionale di filosofia del diritto": pp. 425-449.

${ }^{17}$ Con un Prólogo a la edición castellana, pp. VII-X, scritto da Norberto Bobbio nella primavera del 1987 (il volume è stato pubblicato anche dalla casa editrice Debate, Madrid 1991, 278 pp.). L'edizione italiana è: Bobbio, Norberto, Teoria generale del diritto, Giappichelli, Torino 1993, 297 pp. La Prefazione, pp. VII-10, dell'agosto 1993 coincide solo in parte con quella dell'edizione in spagnolo.

${ }^{18}$ Dispense ciclostilate, senza data sul frontespizio; il colophon indica: “ultimato di stampare il 25 giugno 1958 ”.

${ }^{19}$ Dispense ciclostilate, senza data sul frontespizio; il colophon indica: “ultimato di stampare il 16 settembre 1960 ”.
} 
teoria della norma viene risolta nella teoria dell'ordinamento, seguendo la concezione kelseniana: anzi, dice Bobbio, “forse andando al di là dello stesso Kelsen” (BOBBIO, 1992, p. 9).

I saggi di Bobbio sulla teoria pura del diritto presentano un peculiare andamento cronologico. Bobbio raccolse in un volume i saggi su Kelsen che era andato pubblicando fra il 1954 e il 1986: quel volume si apre infatti con il saggio del 1954 in cui Bobbio analizza i critici di Kelsen (escludendo quindi la già ricordata critica a Carnelutti del 1949), cui seguono però più di dieci anni di silenzio sui temi kelseniani ${ }^{20}$. Nel 1967 un saggio su essere e dover essere segna l’inizio della revisione critica della teoria pura del diritto, cui segue nel 1971 un saggio sulle fonti del diritto in Kelsen. Invece nel 1981-82 si susseguono ben tre saggi sul problema del “potere” nella concezione kelseniana.

Il potere è il tema con cui il Bobbio filosofo della politica ritorna al Bobbio filosofo del diritto $^{21}$ e, nella teoria kelseniana, è un punto cruciale nella separazione fra il mondo della realtà e il mondo della normatività, tra l'essere e il dover essere. Non a caso, quindi, il rapporto fra diritto e potere dà il titolo non solo alla parte centrale, ma anche all’intero volume da Bobbio poi dedicato a Kelsen.

Proprio il tema del potere dimostra come gli interessi giuridici e quelli politici siano sempre stati presenti nel pensiero di Bobbio: già nel 1966 aveva tenuto un corso sul potere dal punto di vista politologico, nell'ambito del Corso di Laurea in Scienze Politiche (che originariamente non era autonomo, ma faceva parte della Facoltà di Giurisprudenza) (BOBBIO, 1966) ${ }^{22}$. Gli ultimi saggi inclusi nel volume del 1992, infine, confrontano il pensiero di Kelsen con quello del sociologo Max Weber e con la teoria dell’argomentazione di Chaïm Perelman. Il volume del 1992 non è quindi unitario - né potrebbe esserlo una raccolta di saggi che si distribuiscono su un arco di più di trent'anni, dal 1954 al 1986 - ma proprio per questo consente di seguire l'evoluzione del pensiero di Bobbio sul positivismo kelseniano.

\footnotetext{
${ }^{20} \mathrm{Nel}$ lungo intervallo tra i due articoli Kelsen è presente nell'insegnamento di Bobbio, in particolare nei due corsi sulla teoria della norma e dell'ordinamento, che nel 1992, quando ormai il suo periodo kelseniano si era concluso da tempo, confluirono poi nel già ricordato volume Teoria generale del diritto.

21 "La teoria giuridica e la teoria politica si integrano e si completano reciprocamente, la prima concentrandosi sul concetto di 'norma' e la seconda su quello di 'potere”” (BOBBIO, 1998, p. 10). "L'ampio e documentatissimo studio" di Greppi (così lo definisce Bobbio stesso) analizza a più riprese "il nesso tra teoria politica e teoria giuridica", tema che non è qui possibile sviluppare. In particolare segnalo le pagine su Elementos del neo-positivismo y de la filosofía analítica durante los años Cinquenta, pp. 93-101.

${ }^{22}$ Bobbio vi distingue la scienza della politica dalla filosofia politica e, fra i numerosi autori citati, viene menzionato non Kelsen, ma Carl Schmitt (p. 75), in anni in cui quest'ultimo era bandito dagli studi sociali e giuridici. Questo fascicolo di "appunti” è meno elaborato delle altre dispense di Bobbio: forse per questo esso non è stato ripubblicato in seguito.
} 


\section{§5. IL DIRITTO COME ORDINAMENTO: UNA TEORIA GENERALE}

Gli studi settoriali di teoria generale del diritto fin qui visti esprimono una visione generale, che però Bobbio non ha mai voluto sistematizzare né in un manuale (“un incubo”, per lui), né in un’opera complessiva. Tuttavia esiste una sua Teoria generale del diritto dalla curiosa genesi colombiana già ricordata nel paragrafo precedente. Pubblicata anche in Italia nel 1993, quando ormai da vent'anni Bobbio si dedicava prevalentemente alla filosofia politica, la sua Prefazione ripercorre la genesi del volume e, in particolare, esprime la riluttanza di Bobbio a presentarsi come autore d'una "teoria generale del diritto": “Accogliendo la recente richiesta di alcuni colleghi ed esaudendo un antico desiderio dell'editore Giappichelli, ho consentito a ripubblicare” in volume unico le due dispense per gli studenti dei corsi del 1957-58 e del 1959-60, rispettivamente sulla norma (BOBBIO, 1958) e sull'ordinamento (BOBBIO, 1960), le quali nel 1987 erano state pubblicate congiuntamente nella traduzione colombiana (BOBBIO, 1987) ${ }^{23}$. I primi passi in questa direzione risalgono però al corso dell'anno accademico 1954-55 e alla relativa dispensa per gli studenti (BOBBIO, 1955b).

Una spiegazione alla riluttanza di Bobbio può forse trovarsi nel fatto che quei corsi erano rivolti agli studenti del primo e del secondo anno, e quindi dovevano ricorrere a semplificazioni che Bobbio accettava nella didattica, ma esitava a trasferire in un libro, che necessariamente si sarebbe rivolto anche agli specialisti. Tuttavia egli stesso, nelle prime righe della parte sulla teoria dell'ordinamento, ricorda che i due corsi degli anni Cinquanta “l'uno e l'altro insieme formano una completa Teoria del diritto, sotto l'aspetto principalmente formale” (p. 159, corsivo di Bobbio).

Leggendo il volume del 1993 si deve anzitutto tener conto che esso riproduce i testi di quelle due dispense degli anni Cinquanta e, quindi, riflette le idee di Bobbio d'una trentina d'anni prima. Nella sua Prefazione del 1993, Bobbio riassume in poche righe la sua intera traiettoria culturale: "Quei due corsi [...] costituiscono la sintesi e, in certo modo, la conclusione del periodo di studi da me dedicato prevalentemente alla teoria del diritto, durante una ventina d'anni che vanno dal primo dopoguerra, in cui compii il mio tirocinio commentando alcuni fra i più noti trattati di teoria generale del diritto e prendendo baldanzosamente le difese di Kelsen contro alcuni suoi detrattori, sino, su per giù, al famigerato '68, quando i predicatori dell'immaginazione al potere rifiutavano sdegnosamente la nuda ragione senza potere, ed io mi avviai, sempre più assiduamente,

\footnotetext{
${ }^{23}$ Con un Prólogo a la edición castellana, pp. VII-X, scritto da Norberto Bobbio nella primavera del 1987. Il volume è stato pubblicato anche dalla casa editrice Debate, Madrid 1991, 278 pp.
}

Revista da Faculdade de Direito - UFPR, Curitiba, vol. 60, n. 3, set./dez. 2015, p. 9-38 
a studi di filosofia politica e passai nel 1972 alla nuova facoltà di scienze politiche per insegnarvi filosofia politica sino all'andata a riposo nel 1979” (BOBBIO, 1993).

Le due dispense e, quindi, il volume del 1993 si collocano nella fase kelseniana di Bobbio, che rivendica puntualmente questa ascendenza. Kelsen è il suo “autore princeps”, né ha "fatto mai mistero", prosegue, “che i due corsi siano d'ispirazione kelseniana”: "kelseniana è, tanto per cominciare, la distinzione fra teoria della norma (singola) e teoria dell'ordinamento (insieme strutturato di norme)” (p. VIII), anche se la definizione di diritto viene ricercata nei caratteri distintivi dell’ordinamento, seguendo "la dottrina italiana dell’istituzione” (p. IX). Kelseniano è ancora l'individuare la caratteristica del diritto nell'ordinamento, e non nella norma, com'era già chiaro "nella distinzione kelseniana tra sistema statico proprio della morale e sistema dinamico proprio del diritto” (p. IX).

Questo volume costituisce il coronamento del pensiero teorico-giuridico di Bobbio e sarebbe quindi temerario tentarne una sintesi: l'esposizione che segue si limita soltanto ad accennare alcuni punti nodali. Data la natura istituzionale del corso da cui prende origine il volume, Bobbio inizia la teoria della norma giuridica esponendo varie teorie del diritto e ricollegandosi così anche agli autori incontrati nel suo saggio sulla consuetudine del 1942, in particolare a Santi Romano e all’istituzionalismo. Proprio quest'ultimo integra la visione normativista di Bobbio, perché la formazione di un gruppo (istituzione) va di pari passo con l'emanazione di regole di condotta: quindi "la teoria dell'istituzione non esclude, bensì include, la teoria normativa del diritto" (p. 14). Inoltre "la teoria dell’istituzione ha avuto il grande merito [...] di mettere in rilievo il fatto che si può parlare di diritto soltanto dove vi sia un complesso di norme formanti un ordinamento, e che pertanto il diritto non è norma, ma un insieme coordinato di norme”, cioè "un sistema normativo" (p. 15).

Bobbio si propone di studiare la norma giuridica dal punto di vista formale, cioè “indipendentemente dal suo contenuto, ovvero nella sua struttura”, intesa come "struttura logicolinguistica”. Si manifesta qui il suo interesse per l'analisi linguistica e per le "questioni di parole” (BOBBIO, 1993, p. IX) ${ }^{24}$, destinato a far scuola negli anni successivi con l'apertura alla filosofia analitica del linguaggio. Bobbio definisce il formalismo giuridico in senso stretto (il diritto non è “ciò che ciascuno deve fare”, bensì la forma, e non il contenuto, di questa prescrizione), distinguendolo dal formalismo etico (“è giusto ciò che è conforme alla legge”) e dal formalismo

\footnotetext{
${ }^{24}$ Poiché la critica di Kelsen alla concezione imperativistica della norma si fonda "su una definizione ristretta di 'comando”, Bobbio commenta: “Ancora una volta si osservi quanta importanza abbiano in queste dispute le definizioni iniziali, e quindi per quanta parte queste questioni siano questioni di parole” (BOBBIO, 1993, p. 106).
} 
scientifico (regole per la costruzione di una scienza giuridica soltanto dichiarativa) (p. 46 s.). Dal punto di vista formale, la norma è una proposizione che può avere una funzione descrittiva, espressiva o prescrittiva. Si apre così il problema del diritto come proposizione prescrittiva e dei rapporti fra le proposizioni prescrittive e quelle descrittive, con le conseguenti distinzioni fra imperativi categorici e ipotetici, fra comandi e consigli. Classificate le norme giuridiche fra i comandi (o imperativi), l'imperatività viene elevata a carattere distintivo del diritto dalle teorie giuridiche che prendono il nome, appunto, di imperativistiche e che vengono respinte da Bobbio.

La norma giuridica è un imperativo rivolto a determinare un comportamento: si pone così il problema del destinatario della norma, che “diventò acuto quando un giurista dell'autorità di Jhering, in polemica con quello che aveva affermato pochi anni innanzi Binding, sostenne che i destinatari della norma giuridica non sono i cittadini, ma gli organi giudiziari incaricati di esercitare il potere coattivo" (p. 93, corsivo di Bobbio). La tesi di Jhering venne accolta anche da Kelsen nella sua distinzione tra norme primarie, rivolte ai cittadini, e secondarie, rivolte agli organi dello Stato. Questa concezione è particolarmente gradita ai giuristi che accentuano il carattere statualistico e coercitivo del diritto. Bobbio invece, seguendo le sue propensioni anche istituzionalistiche, formula al riguardo alcune critiche, poiché gli organi statali non possono essere gli “unici” destinatari della norma: un ordinamento giuridico si rivolge tanto ai cittadini quanto agli organi dello Stato. La tesi di Jhering va dunque temperata, ma contiene "un nucleo di verità” (p. 95).

Le teorie imperativistiche del diritto concepiscono la norma come giudizio ipotetico ("Se ..., allora tu devi”); ma "un giudizio, secondo i dettami della logica classica, non è un comando". Bobbio segue questa corrente, che è in armonia con la propria costruzione: infatti "la dottrina antimperativistica si identifica di solito con la dottrina kelseniana” perché gli argomenti di Kelsen “ad alcuni parvero decisivi” sin dagli Hauptprobleme del 1911 (p. 106). In particolare, la concezione della norma giuridica come giudizio ipotetico permette a Kelsen di distinguere la norma giuridica da quella morale (che è invece comando) e dalla legge naturale, introducendo la differenza tra legge di imputazione (propria del diritto) e legge di causalità (propria del mondo naturale). Bobbio analizza a fondo la posizione di Kelsen, giungendo alla conclusione che "la teoria di Kelsen, per cui la norma giuridica si risolve in un giudizio ipotetico, non è una teoria contraria alla tesi della norma giuridica come prescrizione, perché il giudizio in cui si esprime la norma è pur sempre un giudizio ipotetico prescrittivo e non descrittivo” (p. 109).

Nel determinare i caratteri differenziali della norma giuridica rispetto a quelle di altri ordinamenti, l'attenzione di Bobbio si concentra sulla sanzione, definita come "risposta alla 
violazione” della norma giuridica (p. 123). Però i vari ordinamenti ricorrono a sanzioni diverse: morali (interne e, quindi, scarsamente efficaci), sociali (non istituzionalizzate) e giuridiche, che sono invece esterne ed istituzionalizzate. Queste due qualificazioni caratterizzano la sanzione giuridica come elemento essenziale della norma giuridica. Quelle che vengono dette norme non sanzionate rinviano in realtà ad altre norme sanzionate, spesso in una catena infinita, finché si giunge alla norma ultima, che non è sanzionata, ma che è rispettata per consenso o adesione. Bobbio tiene però distinta questa constatazione storico-empirica ("non vi è Stato tanto dispotico che non faccia assegnamento anche sul consenso”, p. 143) dall'analisi teorica, che invece considera sincronicamente l'ordinamento giuridico preso nel suo complesso (e quindi ammette che esso possa contenere una norma non sanzionata, purché l’ordinamento sia sanzionato nel suo insieme).

All'interno della categoria delle norme giuridicamente sanzionate sono possibili varie distinzioni. Alcune vanno lasciate alle singole discipline giuridiche, come la distinzione tra norme sostanziali e processuali, consuetudinarie e legislative, statuali e internazionali. Dal punto di vista teorico generale, invece, Bobbio distingue le norme generali e singolari, affermative o negative, categoriche o ipotetiche, richiamandosi al fondamento formale della singola distinzione, cioè “esclusivamente alla struttura logica della proposizione prescrittiva” (p. 146, corsivo di Bobbio). L'analisi della norma giuridica si conclude così con argomentazioni logiche desunte dalla distinzione tra proposizioni affermative e negative, "distinzione tradizionale della logica classica, che può essere applicata alle proposizioni giuridiche”, e applicata anche alle norme giuridiche. Qui Bobbio desume dal logico Blanché una serie di schemi grafici, in base ai quali "partendo da una qualsiasi proposizione se ne ottengono altre con il vario uso del segno non” (BOBBIO, 1993, p. $151)^{25}$. Questo complesso esercizio combinatorio conclude l'analisi formale della norma giuridica e permette a Bobbio di passare dall'analisi della struttura della singola norma giuridica all'analisi della struttura dell'insieme di norme giuridiche, organizzate sistematicamente nell'ordinamento giuridico.

Anche l'ordinamento giuridico è analizzato dal punto di vista formale: poiché le norme esistono non isolatamente, ma in insiemi all'interno dei quali sono collegate da specifici rapporti, l'analisi di Bobbio si concentra su questi rapporti. Lo studio della struttura dell'ordinamento giuridico non poteva vantare una tradizione antica come quello della norma giuridica e quindi Bobbio sottolinea “il carattere sperimentale di questo corso". Fino ai teorici dell’istituzione, le

\footnotetext{
${ }^{25}$ Gli schemi logici sono tratti da BLANCHÉ, 1955.
} 
teorie giuridiche avevano considerato la norma come l'elemento primario del diritto, e quindi avevano visto nell'ordinamento "un insieme di tante norme, ma non un oggetto autonomo di studio" (p. 160). Anche se già nel 1917 Santi Romano aveva intitolato il suo libro L'ordinamento giuridico, tuttavia solo con Kelsen è avvenuto “l'isolamento dei problemi dell'ordinamento giuridico da quelli della norma giuridica, e la trattazione autonoma dei primi come una parte di una teoria generale del diritto” (p. 161). Punto di riferimento per Bobbio è la Teoria generale del diritto e dello Stato di Kelsen, pubblicata in Italia nel 1952 (KELSEN, 1952) ${ }^{26}$, che nella parte dedicata al diritto distingue la "Nomostatica” (sulla norma giuridica) dalla "Nomodinamica” (sull'ordinamento giuridico). Questa teoria di Kelsen esercitò un’influenza decisiva su Bobbio, che dichiara: “Il mio corso si riallaccia direttamente all'opera di Kelsen, di cui costituisce ora un commento, ora uno sviluppo" (p. 161).

Nella teoria della norma, Bobbio aveva definito la norma giuridica come caratterizzata dalla sanzione esterna e istituzionalizzata; e proprio quest’ultimo termine indica che, "affinché ci sia diritto, occorre che ci sia, grande o piccola, un'organizzazione, cioè un completo sistema normativo" (p. 166). Anche il sistema normativo viene analizzato dal punto di vista formale, indagando quali sono i rapporti o nessi che collegano fra loro una pluralità di norme, facendone un ordinamento o sistema. La teoria dell'ordinamento interseca così quella dei sistemi e le caratteristiche formali del sistema in generale valgono anche per il sistema giuridico: unità, coerenza e completezza costituiscono perciò le tre caratteristiche dell'ordinamento giuridico in quanto sistema di norme. A ciascuna di queste caratteristiche Bobbio dedica un capitolo della sua esposizione, qui commentata in estrema sintesi.

La teoria dell'unità dell’ordinamento implica una gerarchia delle norme. Si ritorna così alla teoria delle fonti giuridiche, già affrontata anche nella teoria della norma. Però nell'ordinamento le singole norme si distribuiscono su vari livelli: e qui Bobbio recepisce "la teoria della costruzione a gradi dell’ordinamento giuridico, elaborata da Kelsen” per spiegare l' “unità di un ordinamento giuridico complesso” (p. 182). Il commento sulla struttura gerarchica dell'ordinamento conduce Bobbio a discutere il suo fondamento ultimo, cioè la norma fondamentale: norma che gli conferisce unità, ma che è anche oggetto delle critiche più severe. Infatti con questa norma non statuita, ma presupposta, “usciamo dalla teoria del diritto positivo, cui ci siamo sinora attenuti, ed entriamo nella secolare discussione intorno al fondamento o meglio alla giustificazione in senso assoluto del potere” (p. 193). La storicità della visione di Bobbio gli

\footnotetext{
${ }^{26}$ L'originale era stato pubblicato solo in inglese: General Theory of Law and State. Traduzione Anders Wedberg, Harvard University Press, Cambridge (Mass.) 1945, XXXIII-516 pp.
} 
consente questo rinvio alla "Gorgone del potere” come fondamento ultimo (benché extragiuridico) dell’ordinamento giuridico. Questa soluzione è invece preclusa a Kelsen dalla rigida divisione tra essere e dover essere, tra mondo della natura e mondo delle norme, che gli vieta di collocare il fondamento del sistema giuridico (appartenente al mondo del dover essere) nel potere fattuale (appartenente al mondo dell'essere).

La teoria della coerenza implica l’assenza di contraddizioni fra norme, cioè di antinomie. L'unità dell'ordinamento, fin qui esaminata, deve essere anche un'unità sistematica, intendendo per sistema una "totalità ordinata”, i cui enti costitutivi siano "in rapporto di compatibilità fra loro" (p. 201). Ritorna qui la distinzione di Kelsen tra sistema statico, proprio della morale ("le norme sono collegate le une con le altre come le proposizioni in un sistema deduttivo”), e dinamico, proprio del diritto ("le norme derivano le une dalle altre attraverso successive delegazioni di potere”, p. 202). Bobbio dubita che l'ordinamento giuridico possa essere definito un sistema dinamico, perché il criterio formale della delegazione del potere di emanare norme può produrre norme in contrasto fra loro: "ma un ordinamento che ammetta nel suo seno enti in contrasto tra loro si può ancora chiamare 'sistema'?” (p. 204). Per lui, invece, “un ordinamento giuridico costituisce un sistema perché non possono coesistere in esso norme incompatibili” (p. 208).

Il problema della determinazione delle antinomie e dei loro vari tipi viene affrontato da Bobbio secondo criteri logico-formali, ricorrendo alle tavole della verità e agli schemi di Blanché. Il problema della loro soluzione (nel caso che l'antinomia in esame sia solubile) prevede vari criteri (cronologico, gerarchico, della specialità), che però possono entrare in conflitto tra di loro. Insomma, “la coerenza è un’esigenza, ma non una necessità” (p. 239). E Bobbio giunge alla conclusione che "due norme incompatibili, di pari livello e contemporanee, sono entrambe valide", ma non sono entrambe efficaci, "nel senso che l'applicazione dell'una al caso concreto esclude l'applicazione dell'altra” (p. 234). Unico rimedio, in questo caso, è l'abrogazione legislativa di una delle due norme.

Infine, la teoria della completezza implica che l'ordinamento includa "una norma per regolare qualsiasi caso” (p. 237), cioè l'assenza di lacune. La completezza è necessaria se il giudice deve emettere una sentenza su tutti i casi sottopostigli e se è tenuto a giudicarli in base a norme incluse in quell'ordinamento. In assenza di una di queste due condizioni, un ordinamento può esistere anche se incompleto. Il "dogma” della completezza è proprio della concezione statualistica dell'ordinamento, poiché se è diritto solo quello emanato dallo Stato, il giudice non può integrare l'assenza di una norma ricorrendo, per esempio, all'equità. La critica al dogma della completezza per la quale Bobbio si richiama ad Ehrlich (1925) e al Movimento del diritto libero - comporta però 
l'apertura a forme di giudizio che mettono in pericolo la certezza del diritto. In questo contesto ritorna anche un tema già affrontato da Bobbio, quello dell'analogia che, con i principi generali del diritto, offre uno strumento per l'autointegrazione dell'ordinamento lacunoso (p. 265 s).

Le tre caratteristiche fin qui esaminate dell’ordinamento giuridico - unità, coerenza e completezza - nascono da problemi interni dell’ordinamento. L'analisi di Bobbio si conclude con l'esame dei problemi che vengono dall'esterno dell'ordinamento, la cui soluzione è rinviata al diritto internazionale o al diritto ecclesiastico.

I due volumi pubblicati nei primi anni Novanta - Diritto e potere del 1992 e Teoria generale del diritto del 1993 - sembrano porre un suggello finale alla fase kelseniana di Bobbio, di fatto già conclusasi una ventina d’anni prima. Questo punto finale vale però soltanto per il Kelsen teorico del diritto. Infatti già da anni Bobbio aveva trovato nel Kelsen teorico dello Stato e della democrazia anche una fonte del proprio pensiero politologico: "Nella sua teoria dello Stato emergono due temi fondamentali, discutendo i quali, specie negli ultimi anni [anteriori al 1992], ho tratto ispirazione, pur senza prefiggermelo, dal pensiero kelseniano, la democrazia e la pace: la democrazia, intesa come un insieme di regole destinate a permettere a un insieme di individui di prendere decisioni collettive col massimo di consenso; la pace, in favore di quella forma di pacifismo che chiamo 'istituzionale', ovvero, usando una formula tipicamente kelseniana, la pace attraverso il diritto" (BOBBIO, 1992, p. 11 et seq.).

A questo punto il presente discorso su Bobbio si muove in due direzioni diverse: il discorso sul Bobbio teorico del diritto si soffermerà sul suo positivismo giuridico e sulla sua svolta funzionalista (cui sono dedicate le pagine seguenti), mentre invece quello sul Bobbio politologo dovrebbe affrontare i temi della democrazia e della pace: ma questi ultimi temi esulano dall'oggetto del presente scritto e non potranno quindi essere neppure sfiorati.

\section{§6. POSITIVISMO E GIUSNATURALISMO IN NORBERTO BOBBIO}

L’essenza della visione del positivismo giuridico in Bobbio è, a mio avviso, contenuta in tre corsi universitari e in due volumi che raccolgono gli scritti in cui Bobbio si occupò del positivismo giuridico.

I tre corsi ebbero luogo nel 1958, nel 1959 e nel 1960 e ad essi corrispondono le dispense intitolate Teoria della norma giuridica, Teoria dell'ordinamento giuridico (entrambe già ricordate in precedenza e confluite nella Teoria generale del diritto) e, infine, Il positivismo giuridico. Sono 
le tappe di un itinerario che esplora le varie teorie su quei temi e si conclude con l'accettazione critica del positivismo giuridico di Hans Kelsen.

I due volumi sono le raccolte di saggi Giusnaturalismo e positivismo giuridico del 1965 e Dalla struttura alla funzione del 1977: essi costituiscono una riflessione sul positivismo giuridico, che arricchiscono prendendo in considerazione la funzione del diritto.

a) La definizione del positivismo giuridico nei tre corsi.

Nei tre corsi Bobbio ha esplorato "l'esperienza giuridica [come] esperienza normativa" (BOBBIO, 1958, p. 3), il "sistema di norme che costituisce l'ordinamento normativo" (BOBBIO, 1960, p. 3) e, infine, il positivismo giuridico tanto nella sua evoluzione storica, quanto nella sua problematicità teorica, giungendo così "al cuore di questa corrente giuridica”, perché "la teoria dell’ordinamento giuridico [è] il contributo originale del positivismo giuridico alla teoria generale del diritto” (BOBBIO, 1961, p. 253). A questo punto egli dispone del materiale storico e analitico per individuare sette modi per definire il positivismo giuridico (che per ragioni di spazio vengono qui soltanto enunciate):

- dal punto di vista dell'approccio al diritto, il positivismo giuridico prende in esame il diritto come fatto, e non come valore; quindi il diritto è valido non perché ritenuto buono, ma perché prodotto in modo formalmente corretto (teoria formalistica del diritto);

- dal punto di vista della definizione del diritto, il positivismo individua il diritto attraverso l'elemento della coercizione, perché una norma senza sanzione sarebbe inefficace (teoria della coattività del diritto);

- dal punto di vista delle fonti del diritto, il positivismo considera la legislazione come fonte prevalente del diritto, riducendo al minimo la rilevanza della consuetudine (teoria del normativismo legislativo);

- dal punto di vista della teoria della norma giuridica, il positivismo concepisce il diritto come comando, che può essere rivolto ai consociati o ai giudici (teoria imperativistica del diritto);

- dal punto di vista della teoria dell'ordinamento giuridico, il positivismo concepisce il diritto come un sistema completo e coerente, privo cioè di contraddizioni e lacune (teoria sistematica del diritto); - dal punto di vista del metodo scientifico-giuridico, il positivismo limita l'attività del giurista alla pura interpretazione dichiarativa o meccanica della norma, escludendo la funzione creativa del giudice, cioè il judge made law o Richterrecht (teoria dell’interpretazione non creativa); 
- dal punto di vista della soggezione o vincolo alla legge, il positivismo prescrive l'obbedienza stretta o addirittura assoluta alla legge (teoria dell'obbedienza incondizionata). Proprio quest'ultima concezione espose il positivismo all'accusa di aver favorito l'accettazione supina delle norme dettate dalle dittature ${ }^{27}$.

b) La revisione critica del positivismo giuridico nei due volumi.

Accettando una certa schematizzazione si può dire che, negli anni del dopoguerra, al positivismo giuridico accusato di connivenza con le dittature venne contrapposto il giusnaturalismo. Nella letteratura giuridica di quegli anni i temi più frequenti erano senza dubbio la crisi del positivismo giuridico e la rinascita del giusnaturalismo. Anche in Bobbio il tema del positivismo giuridico è sempre collegato a quello del giusnaturalismo, però in una tensione che non si risolve a favore di nessuno dei due, ma che resta presente nella sua coscienza - come in quella di molti altri giuristi di quegli anni - come una lacerazione: da un lato, il positivismo giuridico come espressione dell'esigenza di austerità scientifica e, dall'altro, il giusnaturalismo come esigenza di libertà morale. Dal loro confronto scaturiscono più chiare le caratteristiche dell'uno e dell'altro: “contraria juxta se posita magis elucescunt”.

Nelle due raccolte di saggi Giusnaturalismo e positivismo giuridico del 1965 e Dalla struttura alla funzione del 1977 trova espressione questa crisi del positivismo giuridico (che in Bobbio si accompagna però a una critica del giusnaturalismo di quegli anni) e un crescente interesse per la funzione del diritto nella società. Mentre del funzionalismo si occuperà il prossimo paragrafo, la lacerazione tra positivismo giuridico e giusnaturalismo risulta chiara in due passi tratti da uno scritto poco noto di Bobbio, l'Introduzione a un'opera che non ho mai scritta (BOBBIO, 1962, pP. 7-9) ${ }^{28}$.

Esso si apre con queste parole: "Il contrasto tra giusnaturalismo e positivismo giuridico si presenta, ora come naturale avvicendamento storico di scuole, ora come antitesi tra due concezioni opposte e inconciliabili del diritto, ora come lacerazione intima tra la nostra educazione scientifica e le nostre esigenze morali. Qualsiasi studioso della mia generazione, in un paese come l'Italia, ha

\footnotetext{
${ }^{27}$ Nel dopoguerra, Gustav Radbruch aveva indicato il positivismo come causa dell'asservimento dei giuristi alle dittature; in seguito si comprese che i loro comportamenti rispondevano a condizionamenti sociali anteriori alla dittatura stessa: cfr. LOSANO, 2002b, pp. 194-201.

${ }^{28}$ Introvabile libro fuori commercio scritto dagli “einaudiani” per due "einaudiani”. Queste pagine di Bobbio ritornano nell'Introduzione del marzo 1965 a Giusnaturalismo e positivismo giuridico (pp. 11-13); rispetto all'Introduzione, la Miscellanea contiene un'aggiunta iniziale e una finale, da me riportate in questo paragrafo. Dunque, unendo queste due citazioni al testo dell'Introduzione del 1965 il lettore può ricostruire il testo integrale pubblicato nell'irreperibile Miscellanea del 1962.
} 
vissuto a fondo il contrasto in tutti questi aspetti” (BOBBIO, 1962, p. 7). Gli scritti di Arturo Carlo Jemolo e di Piero Calamandrei, di Guido Fassò e di Giuseppe Capograssi esprimono lo sgomento per l'arbitrarietà delle leggi dittatoriali e il tentativo di superare “l'ingiustizia legale” ricorrendo a un “diritto sovralegale" ${ }^{29}$, cioè al giusnaturalismo.

La posizione di Bobbio non è legata a una scelta di campo, ma alla convinzione che ogni scelta di campo è condizionata dal momento storico che il singolo sta vivendo: "Proprio per aver vissuto a fondo i motivi del contrasto tra positivismo giuridico e giusnaturalismo, non ho mai creduto di potermi schierare recisamente da una parte o dall'altra. Se ho dimostrato simpatie giusnaturalistiche, l'ho fatto nei tempi del positivismo trionfante; così come ho mostrato simpatie positivistiche (dalla difesa del Kelsen sino alla ricerca di argomenti contrari al diritto naturale) in questi ultimi anni di rinnovato giusnaturalismo. Piuttosto che schierarmi da una parte o dall'altra, ho preferito nelle pagine che seguiranno chiarire la complessità dei termini del contrasto, la impossibilità di ridurre il problema dei loro rapporti ad una sola alternativa, e in definitiva mostrare le ragioni per cui lo schierarsi da una parte o dall'altra è spesso più il frutto di una scelta irrazionale che di una meditata riflessione” (BOBBIO, 1962, p. 9, corsivo mio).

"Nelle pagine che seguiranno": Bobbio pensava allora al "libro che non ho mai scritto" e che quindi non avrebbe seguito quella prefazione. In realtà, anche se in forma di raccolta di saggi, quel libro vide la luce pochi anni dopo, nel 1965, in un contesto che permise a Bobbio di riprendere quei temi e di usare come premessa al volume anche la parte centrale di quell'estemporanea e solitaria prefazione.

\section{§7. DAL POSITIVISMO GIURIDICO ALL’APERTURA AL FUNZIONALISMO}

I corsi sulla norma e sull'ordinamento segnano per Bobbio la conclusione dei suoi studi dedicati prevalentemente alla teoria del diritto di stampo kelseniano. Questo periodo intensamente kelseniano va quindi, all’incirca, dal dopoguerra al “famigerato '68”, a partire dal quale gli interessi culturali di Bobbio si spostano dalla filosofia giuridica alla filosofia politica e si concretano anche nel suo passaggio nel 1972 alla Facoltà di Scienze Politiche.

Sino al 1967 Bobbio aveva accettato la concezione kelseniana che distingueva il diritto, da un lato, e la scienza del diritto, dall'altro. Da questa distinzione discende il carattere prescrittivo alle

\footnotetext{
${ }^{29}$ Gustav Radbruch aveva pubblicato l'articolo Gesetzliches Unrecht und übergesetzliches Recht nella "Süddeutsche Juristenzeitung” del 1946; il saggio venne poi ripreso nella sua Rechtsphilosophie curata da Erik Wolf: Koehler, Stuttgart 1956, pp. 347-357.
} 
norme giuridiche e il carattere descrittivo alle proposizioni con cui la scienza giuridica enuncia le norme. Innovando la sua stessa terminologia originaria, Kelsen parlava di "norma giuridica" (Rechtsnorm) nel primo caso e di "proposizione giuridica” (Rechtssatz) nel secondo, e si mantenne sempre fedele a questa distinzione concettuale e terminologica. Bobbio invece nel 1967 si distaccò da questo modello, sostenendo che anche la scienza giuridica di Kelsen aveva un carattere prescrittivo, in quanto diceva al giurista come fare scienza giuridica: gli prescriveva cioè di descrivere (BOBBIO, 1965). Infatti il modello descrittivo kelseniano contiene anche le regole per costruire il modello stesso, e questo spiega la presenza di un linguaggio ora descrittivo ora prescrittivo nella dottrina pura del diritto ${ }^{30}$. Si trattava insomma di indicare dove correva il confine tra realtà e normatività, tra mondo dell'essere e mondo del dover essere, fra i quali - affermava Kelsen - non doveva esserci alcun rapporto.

In quegli anni il tema neokantiano dei rapporti fra essere e dover essere era al centro del dibattito filosofico-giuridico, tanto che ad esso venne dedicato il congresso della "Internationale Vereinigung für Rechts- und Sozialphilosophie” (IVR) del settembre 1967 a Gardone Riviera, dove Bobbio presentò una relazione su essere e dover essere in Kelsen. A quel congresso "noi torinesi” ci eravamo preparati con un convegno sullo stesso tema, nel marzo del $1967^{31}$.

Quel congresso a Gardone fu anche l’occasione di memorabili incontri personali, destinati a durare decenni e a far circolare il pensiero di Bobbio: io conobbi Miguel Reale, mentre Elías Díaz incontrò Bobbio per la prima volta ${ }^{32}$. Tuttavia, indipendentemente da queste occasioni di incontro e di confronto, il positivismo kelseniano era già da tempo al centro degli studi individuali di molti di noi e aveva segnato tanto l'inizio delle nostre carriere accademiche quanto le nostre prime impostazioni teoriche.

Infatti gli anni Sessanta furono, per Bobbio, gli anni dell'approfondimento della teoria pura del diritto e, in particolare, della sua natura sistematica. In questa analisi vennero coinvolti anche gli allievi più vicini, i cui lavori possono essere visti come approfondimenti di singoli aspetti delle sette

\footnotetext{
${ }^{30}$ In quello stesso periodo avevo appena pubblicato la traduzione italiana della seconda edizione della Dottrina pura del diritto e nel 1968 a Parigi, sotto l'influenza di Bobbio, parlai più volte di questo problema con il logico Georges Kalinowski, che aveva analizzato criticamente la relazione di Bobbio al congresso di Gardone (come quest'ultimo ricorda in BOBBIO, 1992, cit., p. 41, nota). Solo dieci anni dopo pubblicai i risultati finali di quegli incontri, che il Maggio parigino aveva interrotto bruscamente: LOSANO, 1978c, pp. 117-151.

${ }^{31}$ All'incontro torinese, cui partecipavano gli allora giovani filosofi del diritto, io presentai la relazione Per un'analisi del "Sollen" in Hans Kelsen, pubblicata poi con gli altri contributi, in "Rivista internazionale di filosofia del diritto", XLIV, 1967, n. 3, pp. 546-568.

32 "Me lo presentò con grande effusione e affetto Renato Treves, il grande amico e maestro, sempre tanto vicino e preoccupato per le persone che venivano dalla Spagna, da quella Spagna che aveva appreso a conoscere e ad amare stringendo amicizia con gli esiliati repubblicani in America Latina, quando egli stesso era lì esiliato dal fascismo italiano" (DÍAZ, 1994, p. 128).
} 
accezioni del positivismo giuridico viste poc'anzi: basti citare il volume di Amedeo G. Conte sulla completezza degli ordinamenti giuridici (1962), lo studio di Giorgio Lazzaro sull'interpretazione sistematica (1965) e il mio volume sulla nozione di sistema e di struttura nel diritto dalle origini fino alla Scuola storica del diritto (1968). Insomma, per usare le parole dello stesso Bobbio, gli anni Sessanta sono quelli dedicati “all’approfondimento dell’analisi strutturale, dal cui grembo fecondo è nata addirittura una disciplina nuova e affascinante, la logica deontica” (BOBBIO, 1977, p. 8) ${ }^{33}$.

Ad essa aggiungerei anche l’informatica giuridica, cui giunsi passando dalla logica formale (la cui astrattezza mi pareva inconciliabile con la funzione pratica del diritto) alla logica della programmazione, che muoveva allora i primi passi. Ma in quegli anni la "cibernetica” era ancora una disciplina oscillante fra le applicazioni pratiche dell'informatica e le costruzioni teoriche della cibernetica sociale. Quest'ultima si adattava bene alla teoria funzionale del diritto: Bobbio riteneva “corretto definire il diritto, dal punto di vista funzionale, come forma di controllo e di direzione sociale”34, rinviando per la "scienza della direzione sociale” (che oggi chiameremmo “management”) a quanto avevo scritto nel 1969 su cibernetica e diritto ${ }^{35}$.

Poiché la teoria di Kelsen è una teoria strutturale del diritto, Bobbio accennava anche all'importanza di esaminare la costruzione di Kelsen alla luce dello strutturalismo, allora in pieno rigoglio soprattutto fra i linguisti e gli antropologi ${ }^{36}$. Per questo propose alla casa editrice Einaudi di tradurre il libro di Raymond Boudon che applicava lo strutturalismo alle scienze umane (BOUDON, 1970). Però, nella cultura francese, le scienze umane non includono il diritto. Perciò non mi limitai a tradurre l'opera, ma la completai indagando che cosa si fosse scritto sullo "strutturalismo giuridico".

I risultati - pubblicati in appendice al volume nel 1970 - dimostravano che i giuristi non andavano oltre all'intenzione di applicare lo strutturalismo al diritto. Poiché non esisteva uno strutturalismo giuridico paragonabile allo strutturalismo linguistico, preferii parlare di “strutturalismo e diritto" (indicando cioè che, in quel momento, i due ambiti di ricerca si

\footnotetext{
${ }^{33}$ La nuova rotta è indicata nel titolo, mentre il sottotitolo distingue questo libro dal precedente volume Studi per una teoria generale del diritto, Giappichelli, Torino 1970, 202 pp.

${ }^{34}$ BOBBIO, 1977, p. 88, nel saggio Verso una teoria funzionalistica del diritto.

35 "Mi pare molto significativo che nelle nuove tendenze della teoria giuridica sovietica il diritto venga compreso nel più vasto ambito della 'scienza della direzione sociale'”, scrive Bobbio, e rinvia al mio Giuscibernetica, Einaudi, Torino 1969, p. 119 et seq. (BOBBIO, 1977, p. 88, n. 28)

${ }^{36}$ Nel 1973 Bobbio scriveva: "La tendenza che nasce con Kelsen verso una teoria del diritto come sistema di norme in relazione interna tra loro non può non trarre illuminanti suggerimenti dal confronto con la svolta saussuriana nella linguistica” (ora in Diritto e potere, p. 78); e poco oltre: “È innegabile che la tendenza di Kelsen a considerare il diritto come un universo strutturato risponde alla stessa esigenza da cui hanno preso le mosse le ricerche strutturali in linguistica e in antropologia” (p. 79).
} 
intersecavano, ma non si fondevano) tanto nel sottotitolo del libro tradotto, quanto nella pubblicazione di quel mio breve scritto nella “Rivista di diritto processuale” (LOSANO, 1970) ${ }^{37}$.

Sistema, struttura, strutturalismo: con il 1970 il giro d'orizzonte sul meccanismo interno dell'ordinamento giuridico poteva dirsi completato. Ma da questa ricognizione le esigenze teoriche di Bobbio non uscivano soddisfatte. Infatti la visione giuridica di Kelsen (come asseriva quest'ultimo) era la continuazione del positivismo giuridico ottocentesco, e questa visione giuridica (come constatava Bobbio) era stata portata da Kelsen a un livello insuperato di affinamento e di perfezione. Intanto però la società che circondava Bobbio e la sua scuola non era più quella dell’Ottocento: perciò dalla metà degli anni Sessanta lo sguardo di Bobbio si andò sempre più distaccando dalla visione puramente strutturale del diritto, cioè dal positivismo normativo di stampo kelseniano.

L’insoddisfazione intellettuale di Bobbio nasceva dalla constatazione che il moderno Stato sociale aveva ormai permeato così a fondo la società che lo stesso diritto, in quanto regolatore di quella società, ne risulta trasformato. In particolare, alla sua funzione repressiva dei comportamenti indesiderati si era andata affiancando in misura crescente una funzione promozionale, che si manifestava negli incentivi con cui lo Stato induce i comportamenti desiderati. Nelle teorie giuridiche strutturali, la funzione del diritto veniva limitata alla minaccia o all'applicazione della sanzione: era lo "Stato castigatore" di Thomas Paine. Nel frattempo lo Stato si era trasformato anche in pagatore e in promotore: la teoria sistematica del diritto non bastava più.

Sempre più spesso negli scritti di Bobbio la concezione sistematica di Kelsen viene contrapposta a quella sociologica del secondo Jhering, in cui la funzione promozionale del diritto ha una posizione di particolare rilievo.

Il crescente interesse per una concezione funzionale del diritto aveva indotto Bobbio a proporre la traduzione dello Scopo nel diritto alla casa editrice Einaudi, che a sua volta l'aveva affidata a me. Più volte avevamo discusso insieme sul termine con cui rendere "Lohnrecht" coniato da Jhering in opposizione a "Strafrecht", diritto penale - e infine ci era sembrato adeguato il neologismo "diritto premiale", che io usai nel testo di Jhering e Bobbio nei suoi saggi sulla funzione del diritto (JHERING, 1970, p. 141; 1972, p. 140) ${ }^{38}$. Questo interesse torinese per le norme premiali

\footnotetext{
${ }^{37}$ Pubblicazione propiziata da Giovanni Conso, che fu mio professore nell'università di Torino. Su questo tema cfr. ora il capitolo Strutturalismo e diritto, in LOSANO, 2002a.

${ }^{38}$ Il termine Lohnrecht è un neologismo anche per Jhering, il quale sottolinea che "il diritto premiale" è un "concetto a noi sconosciuto". Nel volume del 1977 Bobbio, sulla base della traduzione allora recente di Jhering, aggiornò il suo articolo del 1969 citandone appunto la frase "In Roma al diritto penale corrispondeva un diritto premiale" (BOBBIO, 1977, p. 20, nota 11, in cui rinvia alla p. 139 di Lo scopo del diritto).
} 
ebbe anche, a sua volta, una sua "funzione promozionale", in quanto trovò riflesso in una tesi milanese, che seguii per conto di Renato Treves ${ }^{39}$, e in un libro di Alessandra Facchi (1995).

L’accettare la funzione come elemento essenziale del diritto non implica però il rigetto di una sua visione strutturale. Si tratta non di un ripudio, ma di un completamento: la spiegazione strutturale del diritto conserva intatta la sua forza euristica, ma deve essere completata con una spiegazione funzionale del diritto, che in Kelsen manca perché Kelsen aveva seguito con rigore la scelta metodologica di concentrarsi sull'aspetto strutturale del diritto, e non su quello funzionale.

Le due visioni del diritto sono per Bobbio complementari ma ben distinte: "Non credo che vi sia bisogno di insistere sul nesso strettissimo fra teoria strutturale del diritto e punto di vista giuridico, da un lato, e teoria funzionale del diritto e punto di vista sociologico, dall'altro: basti pensare all'espulsione del punto di vista sociologico nella teoria pura del diritto di Kelsen” (BOBBIO, 1977, p. 90). La formulazione è ancora più netta nella prefazione all'edizione colombiana della sua Teoria generale del diritto: "Gli elementi di questo universo [del diritto] messi in luce dall'analisi strutturale sono diversi da quelli che possono esser messi in luce dall'analisi funzionale. I due punti di vista non soltanto sono perfettamente compatibili, ma si integrano anche reciprocamente e in modo sempre utile” (BOBBIO, 1987, p. IX et seq.). Il percorso teorico di Bobbio veniva così a convergere con quello dell'amico Treves, che in quegli anni stava introducendo la sociologia del diritto in Italia.

È ormai tempo di concludere questa sintetica parabola del pensiero giuridico-teorico del Bobbio filosofo del diritto dal positivismo normativo di stampo kelseniano, attraverso un positivismo per così dire critico ${ }^{40}$, fino a una visione tanto strutturale quanto funzionalistica del diritto (e non uso l'aggettivo 'struttural-funzionalistico' perché potrebbe indurre a un indebito accostamento tra Bobbio e Parsons). I chiarimenti apportati da Bobbio anche alla concezione funzionale del diritto additano ancor oggi un ampio campo di indagine tanto ai teorici quanto ai sociologi del diritto.

\footnotetext{
${ }^{39}$ La tesi di Paola Mora, Sanzioni positive, venne presentata da Renato Treves nell'anno accademico 1972-73 alla Facoltà di Giurisprudenza dell'Università Statale di Milano e venne ricordata anche da Bobbio, quando ripubblicò il saggio Le sanzioni positive nella raccolta del 1977. Il riferimento manca ovviamente nell'originale di quel saggio, pubblicato nel 1971 negli Studi dedicati ad Antonio Raselli, Giuffrè, Milano 1971, vol. I, pp. 229-249.

${ }_{40}$ Bobbio stesso definisce "interpretazione critica del positivismo giuridico" la seconda parte - quella appunto dedicata al positivismo - del suo Giusnaturalismo e positivismo giuridico, cit., p. 17 (Premessa).
} 


\section{§8. DAL POSITIVISMO DI BOBBIO A UNA TEORIA “VERAMENTE” GENERALE DEL DIRITTO}

Bobbio con il suo insegnamento mi trasmise così anche la sua eredità sul positivismo giuridico e mi affidò poi la traduzione della seconda edizione della Dottrina pura del diritto. Avevo dunque come formazione di base le idee di Bobbio e di Kelsen, ma i rapporti internazionali e i viaggi mi portarono a contatto con i diritti extraeuropei, dei quali cercai di fornire non una teoria complessiva, ma almeno una descrizione (LOSANO, 1978a) ${ }^{41}$. Mi resi progressivamente conto che il positivismo di origine kelseniana aveva una sua indiscutibile validità, ma che essa era più limitata di quanto a prima vista sembrasse.

In estrema sintesi, la dottrina pura del diritto è la teoria "generale" di un "particolare" diritto che presenta tre caratteristiche: è un diritto codificato (cioè è il diritto europeo-continentale dell'epoca industriale, quindi quella dottrina si adatta meno, per esempio, al Common Law); è un diritto di origine statale (cioè è il diritto di origine democratico-parlamentare: quindi quella dottrina si adatta meno ai diritti storici o a quelli consuetudinari); è un diritto laico, cioè non influenzato da una morale (quindi quella dottrina si adatta meno ai diritti che impongono una morale religiosa, come il diritto islamico, o laica, come le dittature di cui Kelsen discute la natura di "religioni senza Dio” [KELSEN, 2012] $]^{42}$ ).

Tracciando la mappa dei diritti europei ed extraeuropei ho toccato con mano i limiti geografici e temporali della dottrina pura del diritto. Ma, al tempo stesso, si è rafforzata in me la convinzione che la dottrina pura del diritto è lo strumento didattico più efficace per spiegare i diritti codificati, laici e di origine democratico-parlamentare. Invece il tracciare una teoria "veramente generale” del diritto - cioè una teoria che comprenda anche i diritti cui manca una o più d'una di queste tre connotazioni - mi è sempre sembrata un'impresa disperata, perché si raggiungerebbero livelli di astrattezza che renderebbero di fatto irrilevanti le spiegazioni offerte dall'intera costruzione teorica.

Unico criterio per identificare un ordinamento giuridico da includere in una teoria “veramente generale” del diritto sarebbe quello della sua effettività. Con il pericolo che ciò

\footnotetext{
${ }^{41}$ È tradotta in portoghese: Os grandes sistemas jurídicos. Introdução aos sistemas jurídicos europeus e extraeuropeus, Martins Fontes, São Paulo 2007, LVII-677 pp.

${ }^{42}$ Trad. it. Kelsen, Hans, Religione secolare. Una polemica contro l'errata interpretazione della filosofia sociale, della scienza e della politica moderne come "nuove religioni". A cura di Paolo di Lucia e Lorenzo Passerini Glazel, Cortina, Milano 2014, XXXVI-391 pp. Sulla genesi di quest’opera postuma di Kelsen cfr. l'articolo: Losano, Hans Kelsen: il giurista positivo, la politica e la religione (in stampa).
} 
comporta: quello di identificare il diritto con ogni ordinamento normativo che abbia la forza di imporre di fatto il rispetto delle proprie norme.

Di fronte a queste difficoltà, ho preferito descrivere il divenire storico ed i principi fondamentali di ciascuno dei grandi sistemi giuridici esistenti, rinunciando alla costruzione di un livello teorico ancora più elevato ${ }^{43}$. Giungo così a questa insoddisfacente conclusione: la dottrina pura del diritto ereditata da Kelsen e Bobbio è una "teoria generale del diritto" che si rivela non generale; invece la mia visione del diritto è "veramente generale”, ma non la si può indicare con l'impegnativo termine di “teoria”.

\section{RIFERIMENTI BIBLIOGRAFICI}

Albini, Pietro Luigi, Elementi della storia del diritto in Italia dalla fondazione di Roma sino ai nostri tempi e nella monarchia di Savoia in particolare, Mussano, Torino 1847.

$156 \mathrm{pp}$.

Principii di filosofia del diritto per P. L. Albini, Tip. Antonio Spargella, Vigevano 1857,

, Saggio analitico sul diritto e sulla scienza ed istruzione politico-legale. Pietro Vitali, Vigevano 1839, 360 pp.

Balbo, Felice et al., Studi in memoria di Gioele Solari, Edizioni Ramella, Torino 1954, 534 pp.

Blanché, Robert, Opposition et négation, “Revue philosophique”, 1955, pp. 187-217.

Bobbio, Norberto, Autobiografia. A cura di Alberto Papuzzi, Laterza, Roma - Bari 1997.

1977.

, Dalla struttura alla funzione. Nuovi studi di teoria generale del diritto, Comunità, Milano

, Diritto e Potere. Saggi su Kelsen, Edizioni Scientifiche Italiane, Napoli 1992.

, Giusnaturalismo e positivismo giuridico, Comunità. Milano 1965, 241 pp.

, Il positivismo giuridico, Giappichelli, Torino, 1961.

, Il problema del potere. Introduzione al corso di scienza della politica. Lezioni del Prof. Norberto Bobbio raccolte da Iliana Secchieri, Cooperativa Libraria Universitaria Torinese, Torino 1966, 91 pp.

, Introduzione a un'opera che non ho mai scritta, nella Miscellanea per le nozze di Enrico Castelnuovo e Delia Frigessi, Einaudi, Torino 1962.

\footnotetext{
${ }^{43}$ Un timido tentativo in questa direzione è l’ultimo capitolo dei Grandi sistemi giuridici, intitolato Dai diritti positivi alle teorie del diritto, pp. 445-460.
} 
, La filosofia civile di Gioele Solari, Accademia delle Scienze, Torino 1952-53, pp. 409-445.

, La teoria pura del diritto e i suoi critici, "Rivista trimestrale di diritto e procedura civile", 1954, pp. 356-377.

229-249.

, Le sanzioni positive, in: Studi dedicati ad Antonio Raselli, Giuffrè, Milano 1971, vol. I, pp.

Prólogo a la edición española, in Contribución a la teoría del derecho. Edición a cargo de Alfonso Ruiz Miguel, Fernando Torres, Valencia 1980.

, Prólogo, in: Andrea Greppi, Teoría e ideología en el pensamiento político de Bobbio, Marcial Pons, Madrid 1998.

, Struttura e funzione nella teoria del diritto di Kelsen, "Rivista internazionale di filosofia del diritto”, 1973, pp. 187-215.

, Studi per una teoria generale del diritto, Giappichelli, Torino 1970, 202 pp.

, Studi sulla teoria generale del diritto, Giappichelli, Torino 1955a.

, Teoria dell’ordinamento giuridico, Giappichelli, Torino 1960, 218 pp. (litografato).

, Teoria dell'ordinamento giuridico. Lezioni raccolte dagli studenti L. Borgi, C. V. Sarasso, G. Witzel, Giappichelli, Torino 1955b, 242 pp. (litografato).

, Teoria della norma giuridica, Giappichelli, Torino 1958, 245 pp. (litografato).

, Teoría general del derecho, Temis, Bogotá 1987, 269 pp.

, Teoria generale del diritto, Giappichelli, Torino 1993, 297 pp.

Boudon, Raymond, Strutturalismo e scienze umane. Con un'appendice su strutturalismo e diritto di Mario G. Losano, Einaudi, Torino 1970, 212 pp.

Carnelutti, Francesco, Introduzione allo studio del diritto, Foro Italiano, Roma 1943, 88 pp.

, Teoria generale del diritto, Foro Italiano, Roma 1940, pp. 55-57.

, Teoria generale del diritto, Società Editrice del “Foro Italiano”, Roma 1946, VII-388 pp.

1951.

, Teoria generale del diritto. Terza edizione emendata ed ampliata, Foro Italiano, Roma

Díaz, Elías, Norberto Bobbio: una filosofía política para la izquierda, in: Díaz, Los viejos maestros.

La reconstrucción de la razón, Alianza, Madrid 1994.

Ehrlich, Eugen, Die juristische Logik, Mohr, Tübingen 1925, VII-337 pp.

Revista da Faculdade de Direito - UFPR, Curitiba, vol. 60, n. 3, set./dez. 2015, p. 9-38 
Facchi, Alessandra, Diritto e ricompense. Ricostruzione storica di un'idea, Giappichelli, Torino 1995, XIV-198 pp.

Falchi, A., Cesare Civoli. Necrologio, "Rivista italiana di diritto penale”, 1931, n. 3-4, pp. 342-344.

Greppi, Andrea, Teoría e ideología en el pensamiento político de Bobbio, Marcial Pons, Madrid 1998.

Gustav Radbruch, Rechtsphilosophie . Hrsg. von Erik Wolf: Koehler, Stuttgart 1956, pp. 347-357.

Jhering, Rudolf, Der Zweck im Recht. Erster Band, Breitkopf \& Härtel, Wiesbaden 1904, ristampa anastatica: Georg Olms, Hildesheim - New York 1970.

, Lo scopo del diritto, Einaudi, Torino 1972.

Kelsen, Hans, General Theory of Law and State. Traduzione Anders Wedberg, Harvard University Press, Cambridge (Mass.) 1945, XXXIII-516 pp.

Religione secolare. Una polemica contro l'errata interpretazione della filosofia sociale, della scienza e della politica moderne come "nuove religioni". A cura di Paolo di Lucia e Lorenzo Passerini Glazel, Cortina, Milano 2014, XXXVI-391 pp.

, Secular Religion. A Polemic against the Misinterpretation of Modern Social Philosophy, Science and Politics as "New Religions". Edited from the estate of Hans Kelsen by Robert Walter, Clemens Jabloner and Klaus Zeleny, Springer, Vienna 2012, XV-292 pp.

Teoria generale del diritto e dello Stato. Traduzione di Sergio Cotta e Giuseppino Treves, Comunità, Milano 1952, 528 pp.

Losano, Mario G., Alle origini della filosofia del diritto a Torino: Pietro Luigi Albini (1807-1863). Con due documenti sulla collaborazione di Albini con Mittermaier, Accademia delle Scienze, Torino 2013, 104 pp.

, Forma e realtà in Kelsen, Comunità, Milano 1981, pp. 179-212.

I carteggi di Pietro Luigi Albini con Federico Sclopis e Karl Mittermaier (1839-1856). Alle origini della filosofia del diritto a Torino, Accademia delle Scienze, Torino 2014 (in stampa).

, I grandi sistemi giuridici. Introduzione ai diritti europei ed extraeuropei, Einaudi, Torino 1978a, XXIII-361 pp.

Os grandes sistemas jurídicos. Introdução aos sistemas jurídicos europeus e extraeuropeus, Martins Fontes, São Paulo 2007, LVII-677 pp.

, Reine Rechtslehre in Italien, in Der Einfluß der Reinen Rechtslehre auf die Rechtstheorie in verschiedenen Ländern, Manz, Wien 1978b, pp. 151-79.

, Sistema e struttura nel diritto, volume 3: Dal Novecento alla postmodernità, Giuffrè, Milano 2002a, pp. 117-192. 
, Sistema e struttura nel diritto; volume 2: Il Novecento, Giuffrè, Milano 2002b.

, Strutturalismo e scienza giuridica contemporanea, "Rivista di diritto processuale”, XXV, 1970, n. 3, pp. 465-476.

Sulla presenza di un linguaggio ora descrittivo ora prescrittivo nella dottrina pura del diritto, "Materiali per una storia della cultura giuridica. Momenti e figure della teoria generale del diritto", 1978c, VIII, 1, pp. 211-219, ripreso poi in Forma e realtà in Kelsen, Comunità, Milano 1981, pp. 117-151.

, Tra democrazia in crisi e corporativismo in ascesa: il primo libro italiano di Hans Kelsen, in Hans Kelsen - Arnaldo Volpicelli, Parlamentarismo, democrazia e corporativismo. Prefazione e cura di Mario G. Losano, Nino Aragno Editore, Torino 2012, pp. 7-79.

3, pp. 471-517.

Un secolo di filosofia del diritto a Torino: 1872-1972, “Teoria politica”, XXV, 1999, n. 2-

Orecchia, Rinaldo, La filosofia del diritto nelle università italiane. 1900-1965. Saggio di bibliografia, Giuffrè, Milano 1967, XLIII-467 pp.

Plebe, Armando et al., Augusto Guzzo, Edizioni di “Filosofia”, Torino 1964, 127 pp.

Solari, Gioele, La dottrina della giustizia nel sistema della moralità di Augusto Guzzo, "Rivista di filosofia”, 1951, n. 4, pp. 378-398.

La vita e il pensiero civile di Giuseppe Carle, in Memorie della Reale Accademia delle Scienze di Torino, Bocca, Torino 1926, pp. 39-188.

Unzueta, María Ángeles Barrère, La escuela de Bobbio. Reglas y normas en la filosofía jurídica italiana de inspiración analítica, Tecnos, Madrid 1990.

Vico, De uno et universo jurisprincipio et fine uno, Napoli 1720.

\section{POSITIVISM IN THE EVOLUTION OF THE THINKING OF NORBERTO BOBBIO}

ABSTRACT: Through the analysis of the intellectual context and of the texts of the author, this article studies the trajectory of the thinking of Norberto Bobbio and its relations with positivism.

KEYWORDS: Positivism. The thinking of Norberto Bobbio. Legal theory. 